\title{
Dopaminergic neurons show increased low-molecular-mass protein 7 activity induced by 6-hydroxydopamine in vitro and in vivo
}

Ming-Shu Mo ${ }^{1 \dagger}$, Gui-Hua Li ${ }^{1 \dagger}$, Cong-Cong Sun ${ }^{2 \dagger}$, Shu-Xuan Huang ${ }^{1}$, Lei Wei ${ }^{1}$, Li-Min Zhang ${ }^{3}$, Miao-Miao Zhou', Zhuo-Hua Wu', Wen-Yuan Guo ${ }^{1}$, Xin-Ling Yang ${ }^{4}$, Chao-Jun Chen ${ }^{5}$, Shao-Gang Qu ${ }^{6}$, Jian-Xing He ${ }^{7 *}$ and Ping-Yi $X u^{1,4^{*}}$

\begin{abstract}
Background: Abnormal expression of major histocompatibility complex class I (MHC-I) is increased in dopaminergic (DA) neurons in the substantia nigra (SN) in Parkinson's disease (PD). Low-molecular-mass protein 7 ( $\beta 5$ i) is a proteolytic subunit of the immunoproteasome that regulates protein degradation and the MHC pathway in immune cells.

Methods: In this study, we investigated the role of $\beta 5$ i in DA neurons using a 6-hydroxydopamine (6-OHDA) model in vitro and vivo.

Results: We showed that 6-OHDA upregulated $\beta 5 i$ expression in DA neurons in a concentration- and time-dependent manner. Inhibition and downregulation of $\beta 5 i$ induced the expression of glucose-regulated protein (Bip) and exacerbated 6-OHDA neurotoxicity in DA neurons. The inhibition of $\beta 5 i$ further promoted the activation of Caspase 3-related pathways induced by 6-OHDA. $\beta 5 \mathrm{i}$ also activated transporter associated with antigen processing 1 (TAP1) and promoted MHC-I expression on DA neurons.
\end{abstract}

Conclusion: Taken together, our data suggest that $\beta 5 i$ is activated in DA neurons under 6-OHDA treatment and may play a neuroprotective role in PD.

Keywords: Parkinson's disease, 6-hydroxydopamine, Immunoproteasome, TAP1

\section{Background}

Oxidative stress, accumulation of aggregated and misfolded protein aggregates, and neuroinflammation have been suggested to play roles in the pathogenesis of Parkinson's disease (PD) $[1,2]$ These factors impair the ubiquitin-proteasome system (UPS) which is critical for protein metabolic homeostasis [3-5], and they promote the replacement of constitutive proteasome subunits $\beta 1$,

\footnotetext{
* Correspondence: drjianxing.he@gmail.com; pingyixu@sina.com ${ }^{\dagger}$ Ming-Shu Mo, Gui-Hua Li, and Cong-Cong Sun contributed equally to this work.

${ }^{7}$ Department of Thoracic Surgery, First Affiliated Hospital of Guangzhou Medical University, Guangzhou 510120, Guangdong China

'Department of Neurology, First Affiliated Hospital of Guangzhou Medical University, Guangzhou 510120, Guangdong, China

Full list of author information is available at the end of the article
}

$\beta 2$ and $\beta 5$ by the respective immunoproteasome catalytic subunits $\beta 1 \mathrm{i} /$ low-molecular-mass protein 2 (LMP2, PSMB9), $\beta 2 \mathrm{i} /$ multicatalytic endo- peptidase complex-like 1 (MECL1, PSMB10) and $\beta 5$ i (LMP7, PSMB8) [6-8]. The immunoproteasome helps to degrade abnormal proteins, present cleaved peptides as antigens to major histocompatibility complex (MHC) molecules and regulate neuroinflammation $[9,10]$.

Immunoproteasome expression is low in normal young human brains but higher in brain specimens from older normal subjects and Alzheimer's disease (AD) patients [11, 12]. LMP2 knockout mice show classic AD-like symptoms and severe oxidative stress involved in $\mathrm{A} \beta$ aggregation [12, 13]. In Huntington's disease (HD), immunoproteasomes may contribute to the metabolism of huntingtin protein, which is not easily degraded by classical proteasomes [14].

(c) The Author(s). 2018 Open Access This article is distributed under the terms of the Creative Commons Attribution 4.0 International License (http://creativecommons.org/licenses/by/4.0/), which permits unrestricted use, distribution, and 
$\beta 5 \mathrm{i}$ also plays an important role in the regulation of oxidative stress in chronic epilepsy and stroke $[15,16]$. $\beta 5 \mathrm{i}$ expression and changes in proteasomal structure have been found in tyrosine hydroxylase $\left(\mathrm{TH}^{+}\right)$cells in postmortem brains of people with PD-like synucleinopathies such as multiple system atrophy (MSA) and progressive supranuclear palsy (PSP) [17]. $\beta 5 \mathrm{i}$ is known to shape the antigenic repertoire presented on MHC-I. A recent study demonstrated that catecholamine neurons were more responsive to MHC-I expression under $\gamma$-interferon (IFN) treatment and that these neurons were more susceptible to neurotoxicity in neuroinflammatory conditions than in control conditions $[18,19]$. However, whether $\beta 5$ i contributes to DA neuronal neurotoxicity remains unclear. In this study, we further explored the role of $\beta 5 \mathrm{i}$ in the loss of dopaminergic (DA) neurons under 6-hydroxydopamine (6-OHDA) insult in vitro and vivo.

\section{Methods}

\section{Cell culture}

SN4741 cells derived from embryonic substantia nigra and maintained in Dulbecco's-modified Eagle's high-glucose medium (DMEM, Life Technologies, Rockville, MD, USA) supplemented with $10 \%$ fetal calf serum (FCS, Irvine Scientific, Santa Ana, CA, USA), 1\% glucose (Sigma, St. Louis, MO, USA), 1\% penicillin-streptomycin (Gibco ${ }^{\mathrm{Tm}}$, Invitrogen, China) and $2 \mathrm{mmol} / \mathrm{L}$ l-glutamine (Gibco ${ }^{\mathrm{Tm}}$, Invitrogen, China). SN4741 cells were kindly provided by Prof. Qian-Yang of the Fourth Military Medical University [20]. Cells were grown at $37{ }^{\circ} \mathrm{C}$ in $5 \% \mathrm{CO}_{2}$ and subcultured every 3 days as described previously [21].

\section{Cell viability, reactive oxygen species (ROS) and chymotrypsin-like function}

CCK-8 (Dojindo, Kumamoto, Japan) was used to analyze cell viability under different treatment conditions according to the manufacturer's recommendations. Cells were trypsinized, suspended and cultured in 96-well plates at a concentration of $5 \times 10^{3}$ cells/well. Each sample was made in triplicate. The plate contained blank, positive and negative control wells. PR-957 (Selleck, Houston, CA, USA) was used as a selective inhibitor of $\beta 5 \mathrm{i}$ in SN4741 cells [22]. Cells were treated with 6-OHDA or PR-957 overnight or for $48 \mathrm{~h}$, respectively, or with control solution. At different time points, $10 \mu \mathrm{l}$ cell counting kit-8 (CCK-8) (Dojindo, Kumamoto, Japan) solution dissolved in $100 \mu \mathrm{l}$ DMEM (Life Technologies, Rockville, MD, USA) replaced the drug in each well. The incubation continued for another $0.5,1$, or $2 \mathrm{~h}$ at $37{ }^{\circ} \mathrm{C}$ following the manufacturer's instructions. The optical density (OD) value at $450 \mathrm{~nm}$ was measured to calculate cell viability using the formula: cell viability $(\%)=[\mathrm{OD}$ (Sample)-OD (blank control) $] /$ [OD (negative control)-OD (blank control)] by an ELISA microplate reader (ELX800, BioTeK, USA).
Rhodamine 123 (Sigma-Aldrich, St Louis, MO, USA) was used to measure the mitochondrial membrane potential disruption. Cells were suspended and cultured in 6-well plates. After overnight incubation, groups were exposed to 6-OHDA, PR-957 or control solution. Cells were washed 3 times with PBS and reincubated with $100 \mu \mathrm{l}$ DMEM (Life Technologies, Rockville, MD, USA) containing $10 \mu \mathrm{g} / \mathrm{mL}$ rhodamine 123 at $37{ }^{\circ} \mathrm{C}$ for 30 mins. The fluorescence of rhodamine 123 was detected by a fluorescence spectrophotometer (Shimadzu, Matsuyama, Japan, RF5000U) at $490 \mathrm{~nm}$ excitation (Ex) and $520 \mathrm{~nm}$ emission (Em).

2', 7'-Dichlorofluorescin diacetate (DCFH-DA; SigmaAldrich, St Louis, MO, USA) was used to measure ROS level following manufacturer's recommendations. Cells were treated with 6-OHDA or PR-957 at different concentrations and exposure durations. After the cells were washed 3 times with PBS, DCFH-DA diluted in DMEM to $10 \mu \mathrm{M}$ was added and incubated at $37^{\circ} \mathrm{C}$ for $20 \mathrm{~min}$. Cells were washed 3 times with DMEM, and the resultant optical density was measured at $488 \mathrm{~nm}$ excitation and $525 \mathrm{~nm}$ emission by a microplate reader (Spectramax Gemini XS, Molecular Devices, Pennsylvania, USA). The amount of generated ROS was calculated using the formula: [OD (Sample)-OD (Negative control)]/ OD (Negative control).

The chymotrypsin-like activity (CTL) of the immunoproteasome was assayed with Suc-LLVY-AMC [2]. Cells were seeded at a concentration of $1 \times 10^{4}$ cells/well in 96-well plates. Each test was performed in 4 replicates. After treatment with different concentrations of 6-OHDA, cells were harvested and lysed in proteolysis buffer (50 mM Tris- $\mathrm{HCl} \mathrm{pH} 7.4,5 \mathrm{mM} \mathrm{MgCl} 2,1 \mathrm{mM}$ DTT \pm $0.25 \mathrm{mM}$ ATP). Then, $100 \mu \mathrm{l}$ containing $2 \mu \mathrm{g}$ cell lysate was mixed with $50 \mu \mathrm{M}$ Suc-LLVY-AMC (Sigma-Aldrich, St Louis, MO, USA). After $1 \mathrm{~h}$ of equilibration, fluorescence was monitored for $3 \mathrm{~h}$ using a SpectraMax M5 plate reader (Molecular Devices, Pennsylvania, USA, Ex/Em: $370 \mathrm{~nm} / 460 \mathrm{~nm})$.

\section{Overexpression plasmid and shRNA transfection}

The $\beta 5$ i overexpression plasmid was synthesized by GeneCopoeia (Product ID: EX-Mm34282-M29, GeneCopoeia, Guangzhou, China). This sequence was inserted into a p-EZ-M29 vector containing neomycin as a stable selection marker. The insertion was confirmed by sequencing. The mU6 vector contained the mCherryFP gene as a marker to identify transfection efficiency (Product ID: CSHCTR001, GeneCopoeia, Guangzhou, China). PSMB8 was suppressed by specific shRNA in the mU6 vector (Product ID: RSH052242-mU6, GeneCopoeia, Guangzhou, China) with target sequences GGAA TGCAGCCCACTGAATTC, GGAAGGTTCAGATTGA AATGG, GCAGGAAGTTACATTGCTACC and GCCA 
AGGAATGCAGGCTATAC and the hairpin loop sequence TCAAGAG. The mU6-pri vector (Product ID: CSHCTR001-mU6, GeneCopoeia, Guangzhou, China) without the target gene and an empty plasmid were used in the negative control $(\mathrm{NC})$ and mock $(\mathrm{M})$ groups, respectively. First, we detected $\beta 5 \mathrm{i}$ mRNA by qQT-PCR and then confirmed $\beta 5 \mathrm{i}$ protein expression by Western blot.

Transfection was performed based on manufacturer's instructions (Invitrogen, Grand Island, NY, USA). Cells were suspended and seeded in 24-well plates at a 50\% cell density after counting. After $24 \mathrm{~h}$ of culture, transfection was performed as follows. Solution A contained $20 \mathrm{pmol}$ shRNA dissolved in $50 \mu \mathrm{l}$ Opti-MEM without serum, and B solution contained $1 \mu$ lipofectamine 3000 (Invitrogen, Grand Island, NY, USA) dissolved in $50 \mu \mathrm{l}$ Opti-MEM without serum. Solution A and B were mixed and kept at room temperature for $20 \mathrm{~min}$. The culture medium for each well was replaced with $400 \mu \mathrm{l}$ serum-free medium. Cells were incubated in this mixture (serum-free medium containing solutions $\mathrm{A}$ and $\mathrm{B}$ ) for $6 \mathrm{~h}$ for transfection, which was then replaced with serum medium. Transfection efficiency was assessed by fluorescence on the following day.

\section{Partial 6-OHDA lesion and behavioral test}

Forty male Sprague Dawley (SD) rats, ranging from 280 to $300 \mathrm{~g}$ in weight, were bred and maintained in the Specific Pathogen-Free Laboratory Animal Center at Guangzhou Medical University (Guangzhou, China). Weight-matched rats were randomly assigned to four groups: the sham group, 6-OHDA (Sigma-Aldrich, St Louis, MO, USA) group, PR-957 (Adooq Bioscience, CA, USA) group and 6-OHDA plus PR-957 group. Rats were anesthetized with ketamine (10\%) /xylazine (2\%) (Sigma Aldrich, St Louis, MO, USA) and injected with $8 \mu \mathrm{g} 6-\mathrm{OHDA}$ in $4 \mu \mathrm{l}$ solvent [0.9\% $w / v \mathrm{NaCl}$ with $0.1 \%$ ascorbic acid (Sigma-Aldrich, St Louis, MO, USA)] into the left anterior medial bundle (Coordinates: AP: $-4.0 \mathrm{~mm}, \mathrm{ML}:-1.5 \mathrm{~mm}$, DV: $-7.8 \mathrm{~mm}$ ). Animals in the 6-OHDA plus PR-957 group were given the same dose of 6-OHDA followed by $4 \mu \mathrm{l}$ PR-957 (50 nM) injected into the lateral ventricle. The sham group was given the same volume of solvent $[0.9 \% w / v \mathrm{NaCl}$ with $0.1 \%$ ascorbic acid]. At 4 weeks after the 6-OHDA injection, rats were tested in the rotation test. Rotation asymmetry was calculated for $30 \mathrm{~min}$ after intraperitoneal injection of $0.6 \mathrm{mg} / \mathrm{kg}$ apomorphine (Sigma-Aldrich, St Louis, MO, USA) as described previously [23]. All animal studies followed the institutional guidelines for animal experiments of Guangzhou Medical University. All procedures were approved by the Institutional Animal Care and Use Committee of Guangzhou Medical University.

\section{Western blot}

After electrophoresis of proteins from SN4141 cells or the midbrain of rats and blocking with $0.5 \%$ BSA in PBS, the PVDF membranes (Pall Corporation, Pensacola, FL, USA) were incubated with primary antibodies such as anti- $\beta 5 \mathrm{i}$ (1:800, Abcam, Cambridge, MA, USA), anti- $\beta 5$ (1:1000, Abcam, Cambridge, MA, USA) or anti- $\beta$-actin (1:2000, CST, Danvers, MA, USA) at $4{ }^{\circ} \mathrm{C}$ overnight. The primary antibodies were diluted in blocking solution (LI-COR Biosciences, Lincoln, NE, USA). After the membranes were washed, they were incubated with fluorescent-conjugated secondary antibodies (1: 15000; LI-COR Biosciences, Lincoln, NE, USA) for $1 \mathrm{~h}$ in the dark. The Odyssey infrared fluorescence detection system (LI-COR Biosciences, Lincoln, NE, USA) was used for scanning and analysis. For traditional Western blot, secondary antibodies conjugated with horseradish peroxidase (HRP, Santa Cruz Biotechnology, Santa Cruz, CA, USA) and the chemical luminescence detection method (ECL, Pierce Biotechnology, Rockford, IL, USA) were used. Data were scanned and analyzed using the GE 600 system (GE Healthcare, Piscataway, NJ, USA).

Following the protocol used by Goyal et al. [24], $1.0 \times$ $10^{4}$ cells were inoculated in 96-well plates (Corning, Sigma-Aldrich, Dorset, UK) for the in-cell western assay. Cells were cultured in DMEM with 10\% FCS (Irvine Scientific, Santa Ana, CA, USA) for 48 h, which was then replaced with 6-OHDA dissolved in FCS-free DMEM, but the control group was cultured in FCS-free DMEM. Then, each well was washed with PBS and fixed in $4 \%$ formaldehyde for $1 \mathrm{~h}$. Formaldehyde was washed away with PBS, and cells were incubated with $0.1 \%$ Triton X-100 in PBS (3 times, 5 min each). Then, cells were treated with blocking solution (LI-COR Biosciences, Lincoln, NE, USA) and incubated with mouse anti- $\beta 5 \mathrm{i}$ (1:800, Abcam, Cambridge, MA, USA) and rabbit anti- $\beta 5 i$ (1:800, Abcam, Cambridge, MA, USA) overnight at $4{ }^{\circ} \mathrm{C}$. After the cells were washed, fluorescent-conjugated secondary antibodies (LI-COR Biosciences, Lincoln, NE, USA), diluted at 1: 1000 in PBS, were added, and the cells were incubated for $1 \mathrm{~h}$ in the dark at room temperature. Cells were with PBS three times in the dark. Then, plates were imaged on an Odyssey infrared scanner (LI-COR Biosciences, Lincoln, NE, USA).

Immunofluorescence staining and immunohistochemistry Brain tissue was cut at a thickness of $15 \mu \mathrm{m}$ and stored at $-20{ }^{\circ} \mathrm{C}$. Primary antibodies used for immunohistochemistry included mouse monoclonal anti-tyrosine hydroxylase (TH) (1:500, MAB318, Merck Millipore, Billerica, MA, USA), anti- $\beta 5 \mathrm{i}$ (1:500, Abcam, Cambridge, MA, USA) and anti-TAP-1 (1:500, ab10356; Abcam, Cambridge, MA, USA). TAP-1 is a downstream protein that receives peptides provided by the immunoproteasome [25]. After 
overnight incubation with primary antibodies, the tissue or cells were incubated with secondary antibodies such as Cy3-conjugated anti-mouse IgG (1:400, Jackson Immunoresearch laboratory, PA, USA) and/or Alexa 488-conjugated anti-rabbit IgG (1:400, Molecular Probes, Eugene, OR, USA). Images were acquired using a fluorescence microscope (BX51, Olympus, Fujinon, Japan). For immunohistochemistry, the secondary antibody used was a horseradish peroxidase (HRP)-conjugated goat anti-mouse IgG (1:1000, Kangcheng, Shanghai, China). Sections were stained with 3, 3 '-diaminobenzidine (DAB) kits (Wuhan Boster Bioengineering Co., Ltd., Wuhan, China). Images were acquired under a microscope (Olympus AX70; Olympus, Tokyo, Japan). Four images at $200 \times$ magnification were taken, with each image covering an area of the $\mathrm{SN}$ or striatum, and combined into one figure. Images were analyzed by ImageJ software (version 1.45; National Institutes of Health, Bethesda, Maryland, USA).

\section{Fast TH staining and laser capture microdissection (LCM)}

To reduce RNA degradation, we used fast $\mathrm{TH}$ staining to detect DA neurons. Slices were fixed in acetonemethanol solution at $-20{ }^{\circ} \mathrm{C}$ for $10 \mathrm{~min}$, washed with PBS containing $1 \%$ Triton X-100, incubated with the $\mathrm{TH}$ antibody (MAB318, Merck Millipore, Billerica, MA, USA) at a 1:100 dilution for $10 \mathrm{~min}$, rinsed in PBS with Triton twice, and incubated with the goat antirabbit antibody with HRP (1:100, Kangcheng, Shanghai, China) for $5 \mathrm{~min}$. Immunohistochemistry staining was done by DAB kits (Wuhan Boster Bioengineering Co., Ltd., Wuhan, China). The stained slices were dehydrated in RNase-free solutions as follows: $100 \%$ acetone for $5 \mathrm{~min}$, 75\% ethanol, 95 and 100\% ethanol for $1 \mathrm{~min}$ each, and then xylene twice for $1 \mathrm{~min}$ and $5 \mathrm{~min}$.

As described previously [26, 27], nonfixed fresh brain tissue was rapidly frozen and cut into $8-\mu \mathrm{m}$-thick slices. Slices were collected on to polyethylene naphthalate membrane-coated glass slides (Life Technologies, Grand Island, NY, USA). After fast tyrosine hydroxylase staining, $\mathrm{TH}^{+}$neurons in the substantia nigra were captured by the Arcturus XT system (Life Technologies, CA, USA). Laser power was set at $70 \mathrm{~mW}$ and $150 \mathrm{mV}$. Approximately 300-450 $\mathrm{TH}^{+}$neurons were collected, and total RNA was extracted using the mirVana PARIS Kit (PN AM1556, Austin, TX, Ambion, USA) and converted into cDNA by a Reverse Transcription Kit (Takara, Shiga, Japan). RT1A (rat monomorphic MHC class I antigen) binds the peptide or antigens translocated by TAP into the ER, and its mRNA level in DA neurons was detected by qRT-PCR. The PCR primers (TIANGEN Biotech, China) used were as follows: GAPDH-F: 5'-TACTAGCGGTTTTACGGGCG-3' and GAPDH-R: 5'-TCG-AACAGGAGGAGCAGAGAGCGA-3';

TAP-1-F: 5'-GGCAGACTCAGTTC-CTCTCAC-3' and
TAP-1-R: 5'-CAGAACGGGTTGGGGATCAA-3'; RT1A (Rat monomorphic MHC class I antigen) -F: 5'-GCTC ACACTCGCTGCGGTAT-3' and RT1A-R: 5'-GCCA TACATCTCCTGGATGG-3'. GAPDH was used as an internal control, and mRNA expression was analyzed using the $2^{-\triangle \Delta C T}$ method [28].

\section{Statistical analysis}

All experiments were repeated at least 3 times. Data are shown as the mean \pm SD. ANOVA was followed by Tukey's or Student-Newman-Keuls (SNK) post hoc testing. $P<0.05$ was considered statistically significant. All analyses were performed using SPSS.13 and STATA software (Version 14; StataCorp, College Station, TX, USA).

\section{Results}

6-Hydroxydopamine upregulates immunoproteasome expression in DA neurons

SN4741 cells were treated with different concentrations of 6-OHDA for $24 \mathrm{~h}$. The in-cell western assay showed that $\beta 5$ was upregulated upon treatment with 6-OHDA for 3-18 $\mathrm{h}$ and then downregulated after $24 \mathrm{~h}$, whereas $\beta 5 \mathrm{i}$ was upregulated by 100-300 nM 6-OHDA for $24 \mathrm{~h}$ (Fig. 1a, b). The Western blot data further confirmed that $\beta 5$ and $\beta 5 \mathrm{i}$ expression were dose-dependently upregulated when the concentration of 6-OHDA was higher than $50 \mathrm{nM}$ (Fig. 1c, d). In the antigen presentation pathway, the expression of glucose-regulated protein (Bip), a regulator involved in protein translocation into the ER [29], and TAP1, a transporter associated with antigen presentation [30], was increased as a dose-dependent manner under 6-OHDA treatment for $24 \mathrm{~h}$ (Fig. 1e, f). In the apoptosis pathway, the expression of proapoptotic proteins, such as Bax, Caspase-3 and cleaved-Caspase-3, was upregulated, and the expression of antiapoptotic proteins, such as Bcl-2, was downregulated significantly by 6-OHDA treatment for $24 \mathrm{~h}$ (Fig. 1g, h).

When SN4741 cells were treated with 200 nM 6-OHDA for different exposure times, $\beta 5$ was upregulated, reaching a peak expression at $12 \mathrm{~h}$ (Additional file 1: Figure S1. A, B), whereas $\beta 5 i$, TAP1 and Bip expression were significantly increased upon 6-OHDA treatment at $24 \mathrm{~h}$ (Additional file 1: Figure S1. A-D). In vivo, increased expression of $\beta 5, \beta 5 \mathrm{i}$, TAP1 and Bip was further confirmed in the $\mathrm{SN}$ of the rat after 6-OHDA treatment for $24 \mathrm{~h}$ (Fig. 2a-d) and was accompanied by an increased percent of neurons with $\beta 5 \mathrm{i}$ and $\mathrm{TH}^{+}$expression (Fig. 2e).

\section{$\beta 5 \mathrm{i}$ dysfunction inhibits antigen presentation in DA neurons}

SN4741 cells were then treated with 50 nM PR-957 [22]. We noted that CTL associated with $\beta 5 \mathrm{i}$ function decreased more than $50 \%$ but had no significant neurotoxicity in vitro 


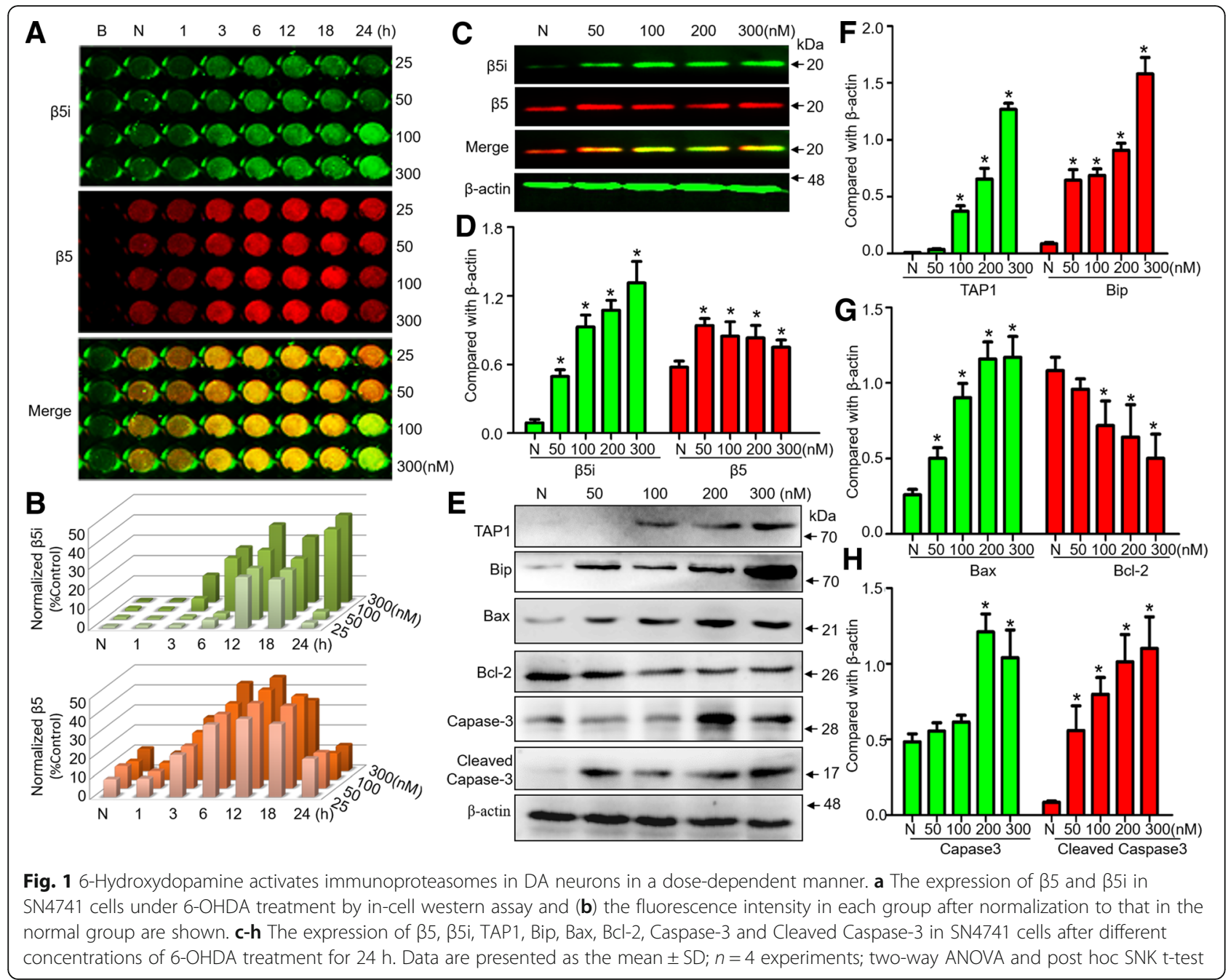

(Fig. 3a). TAP1 expression was decreased after $50 \mathrm{nM}$ PR-957 treatment for $3 \mathrm{~h}$ (Fig. 3b, c). PR-957 at $100 \mathrm{nM}$ induced neurotoxicity, with significant upregulation of Bax and Bip and downregulation of $\mathrm{TH}$ after $24 \mathrm{~h}$ treatment (Fig. 3d-f). In vivo, 50 nM PR-957 was stereotaxically injected into the left anterior medial bundle in SD rats. After $24 \mathrm{~h}, \mathrm{TAP} 1$ and Bip expression in the lesioned side of the SN significantly decreased and increased, respectively (Fig. 3g-h), but TH expression was not changed (Fig. 3i). Our data suggested that 50 nM PR-957 for 24 h may be optimal for $\beta 5 \mathrm{i}$ inhibition without significant neurotoxicity in vitro and in vivo.

\section{$\beta 5 \mathrm{i}$ inhibition exacerbates 6-hydroxydopamine-induced DA neuronal damage}

Cell morphology, viability and apoptosis were not significantly affected by treatment with PR-957 alone in vitro. However, cotreatment with PR-957 and 6-OHDA dramatically induced cell shrinkage and pyknosis, accompanied by a significant increase in ROS and decrease in cell viability compared to treatment with 6-OHDA alone (Fig. 4a, b). Treatment with PR-957 alone did not affect Caspase- 3 or cleaved Caspase- 3 expression, but cotreatment significantly exacerbated the 6-OHDA-induced activation of Caspase-3 and cleaved Caspase-3 (Fig. 4c, d). In vivo, we examined the additional loss of DA neurons in the 6-OHDA hemilesioned rats after inhibition of $\beta 5 \mathrm{i}$ by PR-957 4 weeks after treatment. The number of $\mathrm{TH}^{+}$cells in the lesioned side did not change after treatment with PR-957 alone. The 6-OHDA-induced hemilesion in the $\mathrm{SN}$ was partial in this 6-OHDA model. Compared to $58.3 \%$ loss of $\mathrm{TH}^{+}$cells under 6-OHDA treatment alone, a $74.2 \%$ loss of $\mathrm{TH}^{+}$cells was observed in the lesioned side of rats with DA neuronal damage exacerbated by PR-957, and these rats also exhibited a significant increase in apomorphine-induced rotation (Fig. $4 \mathrm{e}-\mathrm{g}$ ). In the striatum, the $\mathrm{TH}$ level on the lesioned side was only at $13.8 \%$ of that 


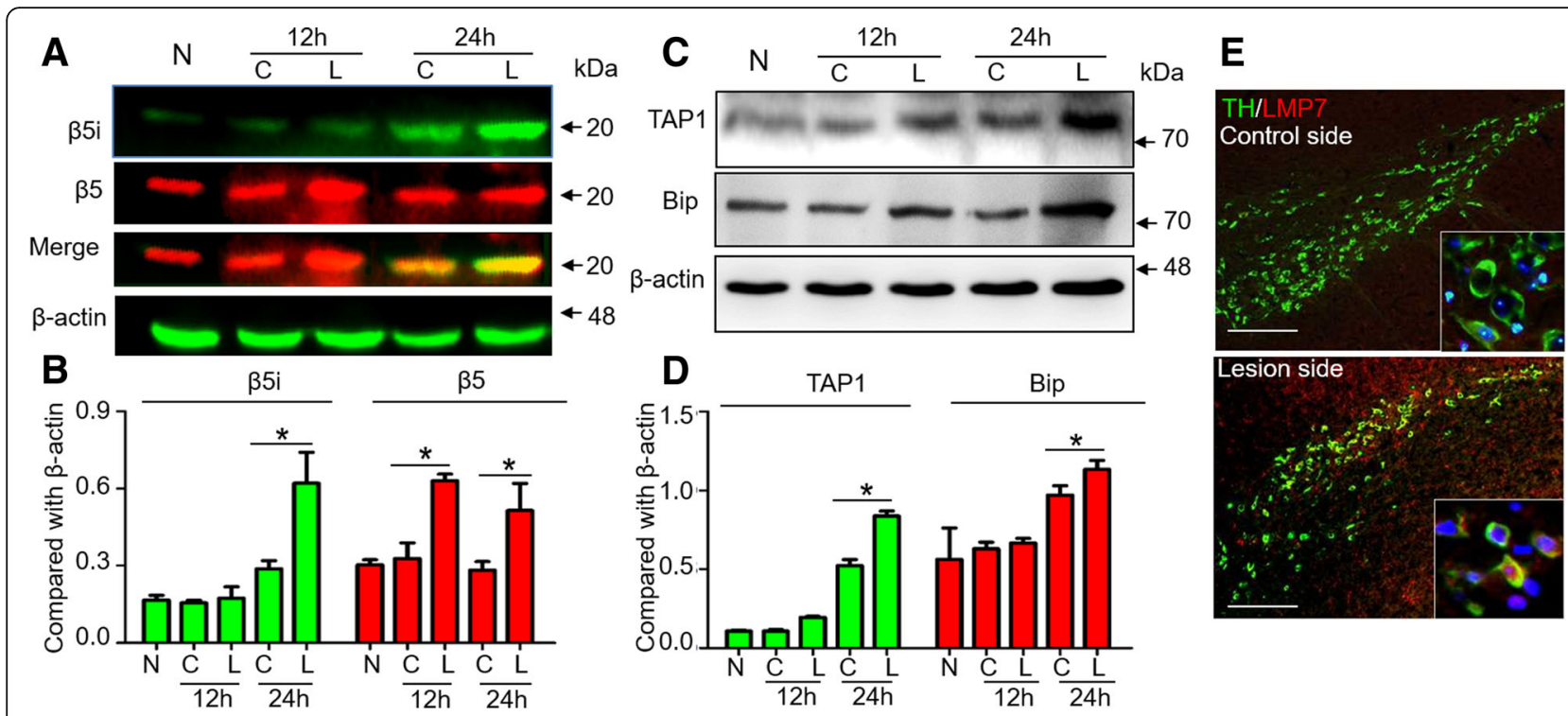

Fig. 2 6-Hydroxydopamine activates immunoproteasomes in DA neurons in vivo. The expression of $\beta 5$, $\beta 5 \mathrm{i}(\mathbf{a}, \mathbf{b})$, TAP1 and Bip (c, $\mathbf{d}$ ) in the SN of the 6-OHDA hemilesioned rat model. e The colocalization of $\beta 5 \mathrm{i}$ on TH neurons in the SN at 1 day after 6-OHDA treatment. Scale bar $=100 \mu \mathrm{m}$. * $P<0.05$, compared with control. N, normal group without treatment. C, control side of the brain. L, lesioned side of the brain. Data are presented as the mean $\pm S D ; n=4$ experiments; one-way ANOVA and post hoc SNK t-test

on the control side in the cotreatment group, which was significantly lower than the $21.5 \%$ in the group given 6-OHDA treatment alone (Additional file 2: Figure S2).

\section{Downregulated $\beta 5 \mathrm{i}$ expression impairs antigen presentation in DA neurons under 6-OHDA treatment} CTL function was significantly impaired and enhanced in SN4741 cells after $\beta 5 \mathrm{i}$ mRNA down- and upregulation, respectively (Fig. 5a). $\beta 5 \mathrm{i}$ expression was manipulated by RNAi in the normal condition (Fig. 5b, c) and with 6-OHDA treatment (Fig. 5d, e). In vitro, the upregulation of $\beta 5 \mathrm{i}$ mRNA significantly promoted TAP1 expression and inhibited Bip expression under 6-OHDA treatment, and downregulation of $\beta 5 \mathrm{i}$ mRNA had an inverse effect (Fig. 5f, g). After $\beta 5 \mathrm{i}$ function was inhibited by PR-957, the 6-OHDA-induced upregulation of TAP1 was significantly inhibited, and Bip expression was slightly increased in SN4741 cells (Fig. 5h, i) and rats (Fig. 6b, c). Immunofluorescence result showed that the 6-OHDA-induced increase in TAP1 expression was colocalized with $\mathrm{TH}^{+}$cells and was attenuated by PR-957 (Fig. 6a). Laser capture microscopy was used to capture $\mathrm{TH}^{+}$neurons from the $\mathrm{SN}$ of rats administered 6-OHDA (Fig. 6d). A significant increase in TAP1 and RT1A (MHC-I) mRNA expression in laser-captured $\mathrm{TH}^{+}$neurons was found at $24 \mathrm{~h}$ after 6-OHDA treatment, which was inhibited by PR-957 (Fig. 6e).

\section{Discussion}

Immunoproteasome and $\mathrm{MHC}$ molecules are minimally expressed in the healthy brain, and their activation and upregulation are indicative of a pathological status in the central nervous system (CNS) [12, 19, 31, 32]. The examination of brain specimens from people with neurodegenerative disorders such as $\mathrm{AD}, \mathrm{HD}$, and amyotrophic lateral sclerosis (ALS) have revealed dysfunctions in immunoproteasome activity [12, 33, 34], and these phenomena have also been found in those with autoimmune encephalomyelitis (EAE) and epilepsy [8, 15]. A similar failure in proteolytic mechanisms, as well as increases in oxidative stress and neuroinflammation, have also been reported in PD [35]. We propose the following scenario to describe the role of $\beta 5 \mathrm{i}$ in PD pathogenesis. Initially, ROS and the unfolded protein response (UPR) induce ER stress and neuronal damage in DA neurons. Then, Bip is activated and ships abnormal proteins from the ER to the UPS. The overloaded proteins induce the activation of $\beta 5 \mathrm{i}$ and transformation of the immunoproteasome to have an updated and expanded proteasome capacity. Consequently, the ubiquitinated proteins are degraded to peptides by $\beta 5 \mathrm{i}$ and recognized by MHC-I. Here, our study revealed that $\beta 5 \mathrm{i}$ is activated in DA neurons exposed to $6-O H D A$, and upregulation of $\beta 5 \mathrm{i}$ or other immunoproteasome components might play a neuroprotective role against ROS-mediated damage in PD.

The UPS is a key factor in the proteostasis network [36]. Different subunit configurations of the UPS, such as the 


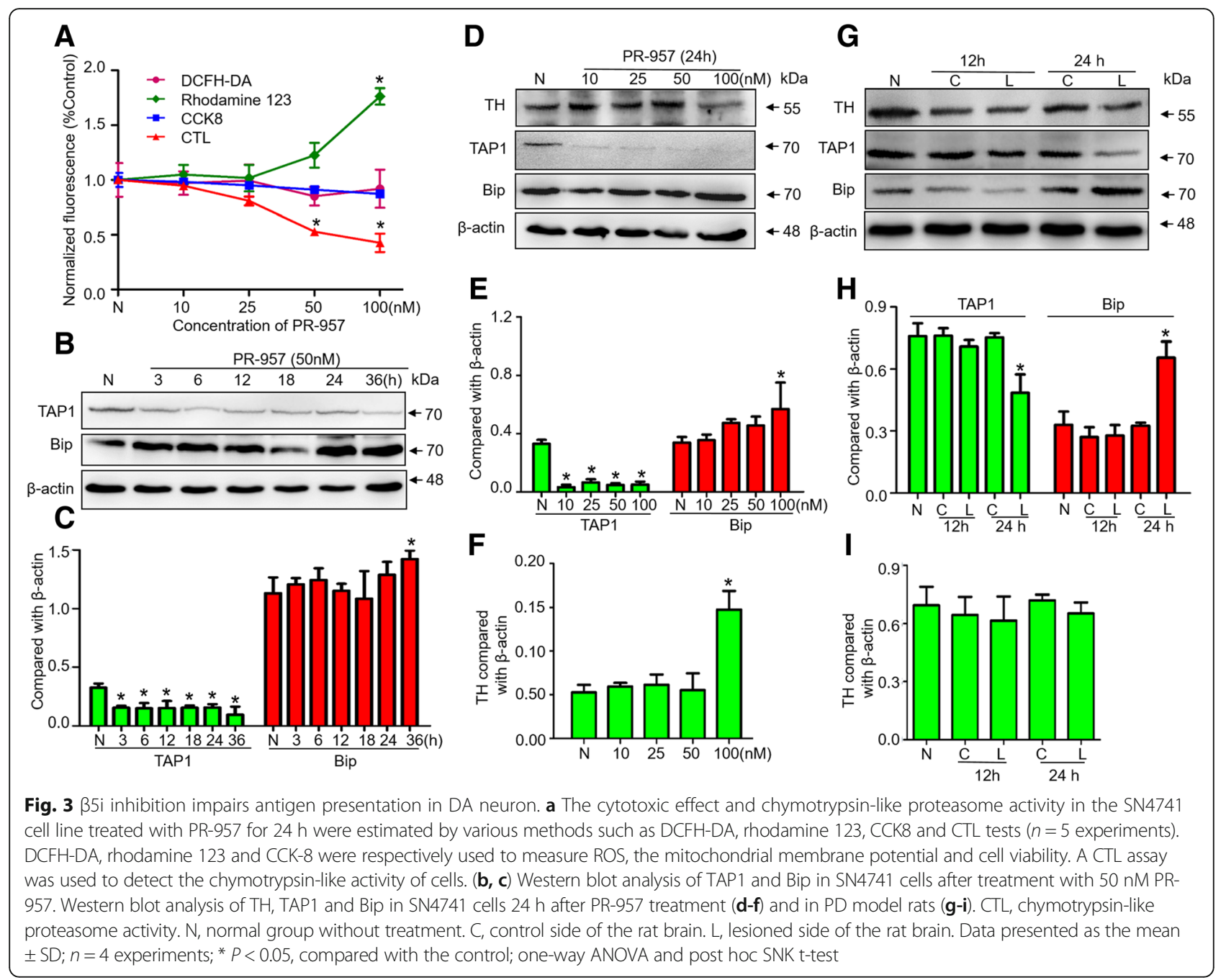

standard proteasome, immuneoproteasome, mixed-type proteasome, thymoproteasome and spermatoproteasome, acquire different proteolytic capacities [37]. The immunoproteasomes activated by ROS, lipopolysaccharide (LPS) and IFN- $\gamma$ have strong capabilities to clear protein deposits and alleviate ROS impairment $[8,38]$. The overloading of damaged proteins and insufficient proteolytic capacity may trigger immunoproteasomes to replace impaired proteasome subunits [39]. X-ray crystallography studies have shown that the enzyme active center of the immunoproteasome (iUPS) provides a larger space than the enzyme active center of other proteasomes to accommodate and degrade misfolded or oxidized proteins [40]. For example, the immunoproteasome eliminates the extended huntingtin proteins of $\mathrm{HD}, \mathrm{A} \beta$ aggregates of $\mathrm{AD}$ and mutant SOD1 deposition of ALS more efficiently [14, 41, 42]. Under normal conditions, oxidized cytoplasmic and nuclear proteins are generally degraded by the proteasome [43]. The $20 \mathrm{~S}$ proteasome, immunoproteasome and PA28 $\alpha \beta$ regulator are all upregulated under $\mathrm{H}_{2} \mathrm{O}_{2}$-induced oxidative stress, and the immunoproteasome may degrade oxidized proteins more selectively than the other proteasomes [43]. Some studies have suggested that the enhanced proteolytic activity of the immunoproteasome more efficiently clears aggregated proteins and is important for cell viability under IFN- $\gamma$ treatment [8]. Others have suggested that the function of the immunoproteasome to bind and degrade ubiquitin conjugates is similar to that of constitutive proteasomes [44]. Recently, we reported that Chinese females carrying the rs17587-G/G mutation of PSMB9 are at a higher risk of PD [35]. The rs17587 variation at exon 4 of PSMB9 affects the glutamyl peptide hydrolyzing activity associated with proteolytic function [45]. As an immunoproteasome subunit, $\beta 5 i$ has been found to be involved in proteinopathies and the innate immune response [37]. In this study, we further explored the role of $\beta 5 \mathrm{i}$ in the 6-OHDA model of PD. Our results showed that $\beta 5 \mathrm{i}$ was activated and upregulated in a 


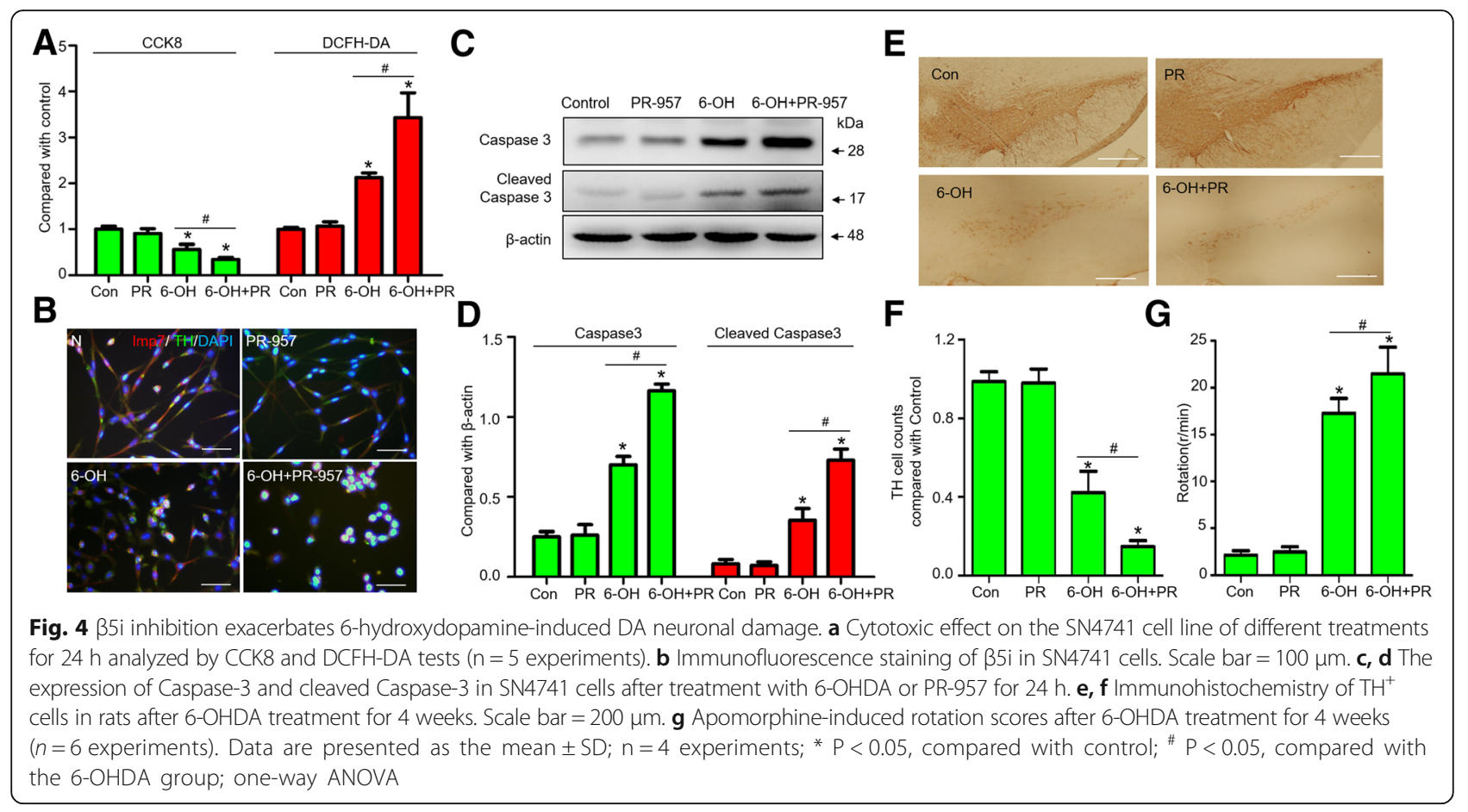

dose- and time-dependent manner after 6-OHDA treatment in a DA neuron cell line, and this was further confirmed in the 6-OHDA hemilesioned rat model of PD. ER stress and oxidative stress have been suggested to contribute to the loss of DA neurons in PD [46]. Compared to the standard proteasome, the immunoproteasome is thought to be more resistant to oxidative stress and ER stress [8]. When protein homeostasis is impaired in neurons, misfolded proteins aggregate in the ER and induce ER stress [47]. Bip is upregulated and binds aggregated proteins for transportation from the ER to the UPS [29]. If the UPS and immunoproteasome system are deficient, neurons are more susceptible to apoptosis due to the stress from the accumulation of oxidized proteins [8, 48]. In aging-related sporadic inclusion body myositis, intracellular protein aggregation was accompanied by ER stress and proteasome dysfunction [49]. A study from X-linked adrenoleukodystrophy revealed that $\beta 5 \mathrm{i}$ was significantly elevated and recruited to mitochondria in response to oxidative stress where it participated in mitochondrial protein quality control [3]. Recently, IFN- $\gamma$-induced oxidative stress was found to upregulate $\beta 5 \mathrm{i}$ expression with increased poly-Ub substrate degradation efficiency [8]. In this study, we used a 6-OHDA model to induce massive oxidative stress and the unfolded protein response in DA neurons [50]. ROS and ER stress occurred in a dose- and time-dependent manner following 6-OHDA treatment [51]. We found that inhibition and downregulation of $\beta 5 \mathrm{i}$ resulted in DA neurons with increased sensitivity to
6-OHDA toxicity, suggesting that the neuroprotective effect of $\beta 5 \mathrm{i}$ may be related to ROS regulation and ER stress at the early stage of PD.

Immunoproteasomes still play an important role in the regulation of neuroinflammation $[13,52]$. In the peripheral immune system, immunoproteasome subunits degrade proteins to peptides, which present to TAP1 as antigens [25]. As a peptide transporter protein, TAP1 loads antigenic peptides into the ER where MHC molecules recognize antigens and present them to the cell membrane $[30,53]$. IFN- $\gamma$-signaling has been proven to promote $\mathrm{MHC}$ class I antigen presentation, and IFN- $\gamma$-regulated inflammation in proteasome-associated autoinflammatory syndromes (PRAAS) was partly reduced after inhibition of proteolytic function [54, 55]. As a highly selective inhibitor of $\beta 5 \mathrm{i}, \mathrm{PR}-957$ was shown to reduce the release of IL-23 and TNF- $\alpha$ from inflammatory cells by 90 and $50 \%$, respectively [22]. PR-957 also inhibits inflammation in MOG35-55-induced experimental autoimmune encephalomyelitis [56]. Notably, neurodegenerative diseases predominantly display disorders of neuroinflammation. In transgenic mouse models of $\mathrm{AD}$ and human postmortem tissue, immunoproteasome activities and HLA-DR expression are strongly increased and accompanied by overactivated microglia in the cortex. $[57,58]$ Previously, neurons were considered to be 'immunoprivileged' without antigen presentation capabilities [59, 60]; now MHC-1 expression has been demonstrated on DA neurons in 


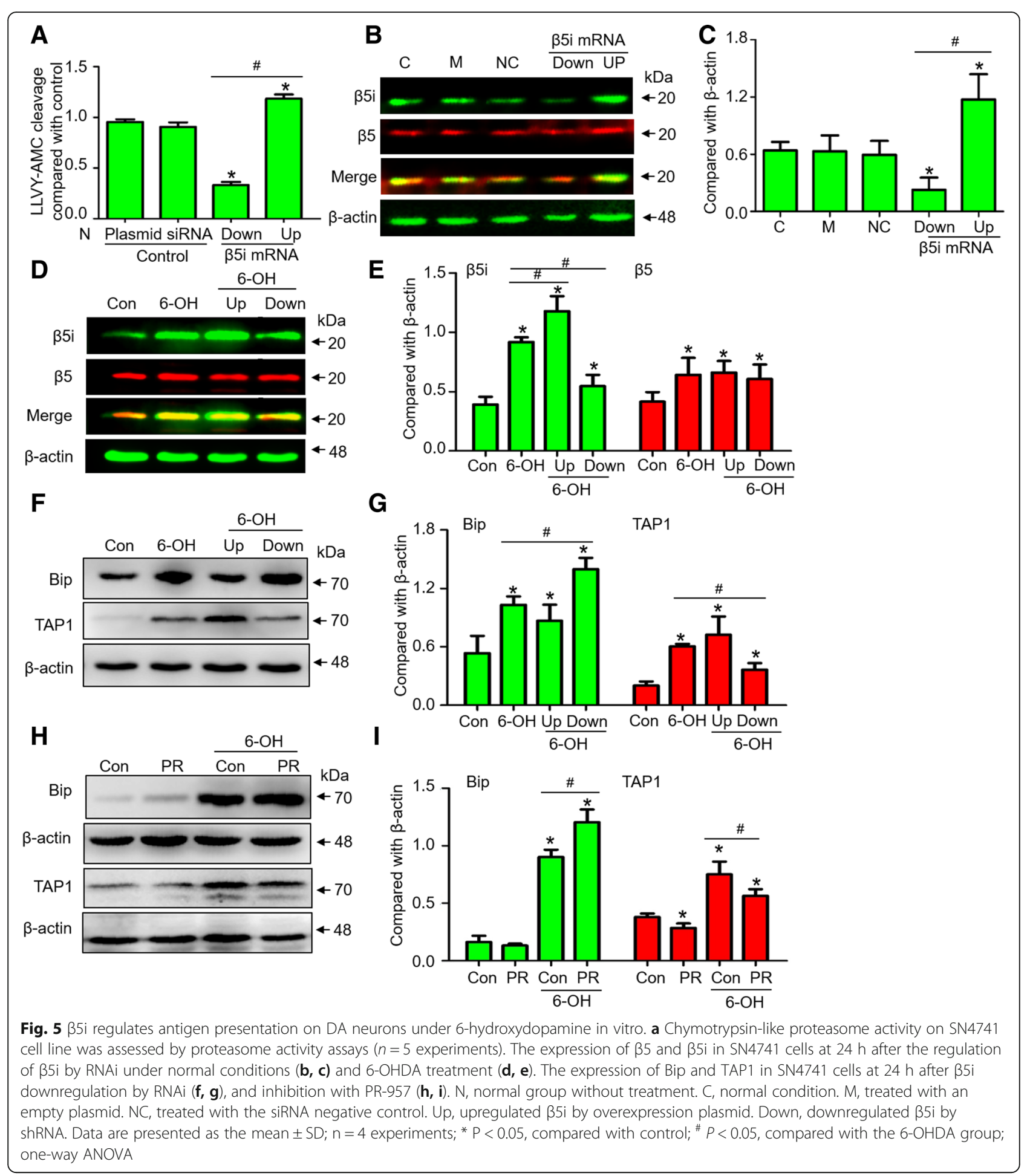

the rodent and human brain [18, 19]. The catecholaminergic neurons expressing MHC-1 have been shown to be more susceptible to apoptosis induction, suggesting that these neurons may be targeted by ROS during the development of PD [19]. In this study, our results revealed significant upregulation of
MHC-I and TAP1 accompanied by increased expression of $\beta 5 \mathrm{i}$ on DA neurons under 6-OHDA treatment and that MHC-I and TAP1 mRNA levels were decreased after $\beta 5 \mathrm{i}$ inhibition. These findings suggest that $\beta 5 \mathrm{i}$ may regulate the TAP1/MHC-I pathway in DA neurons under oxidative stress. 


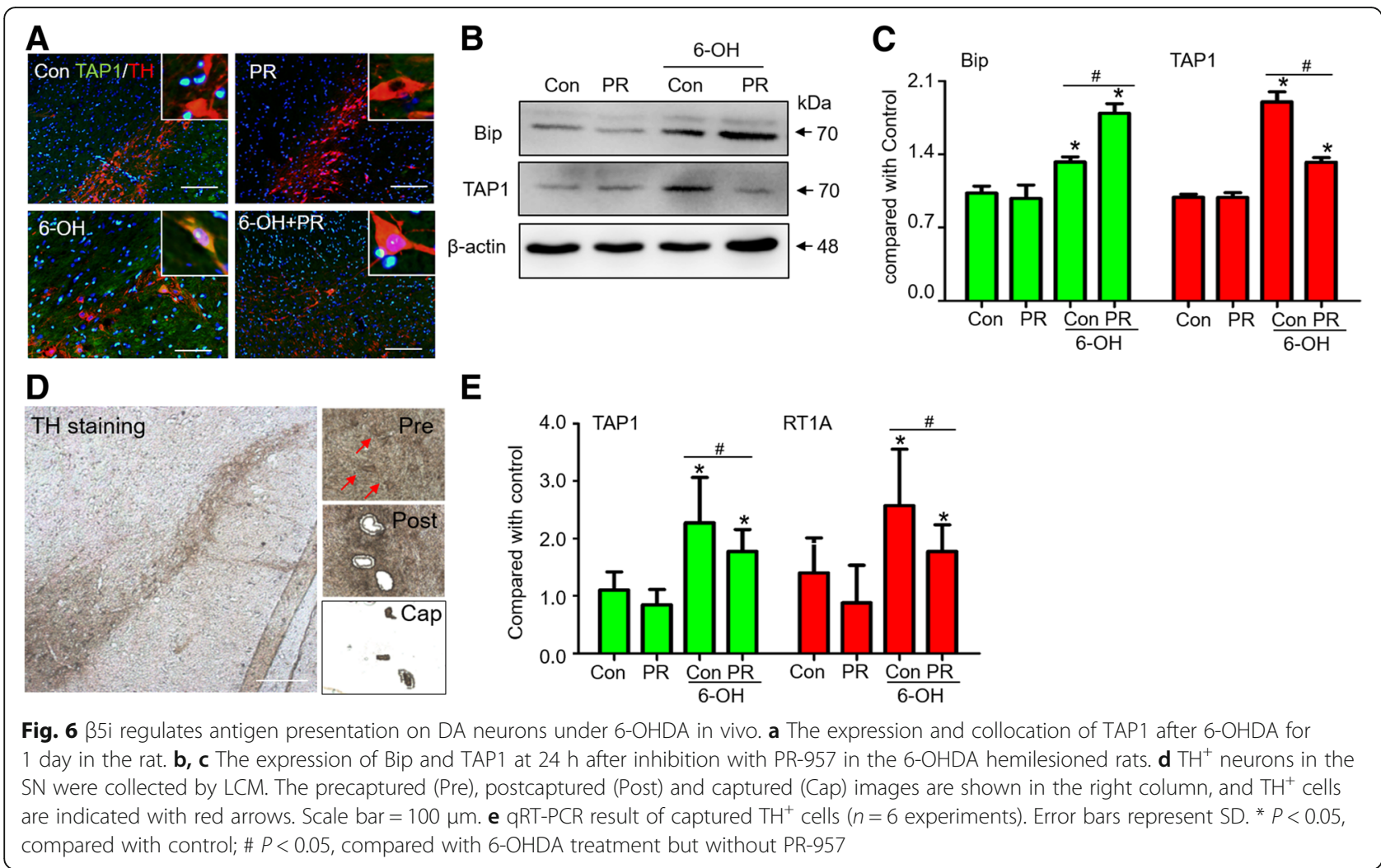

\section{Conclusions}

In conclusion, our data showed that $\beta 5 \mathrm{i}$ was activated by 6-OHDA-induced oxidative stress in DA neurons both in vitro and in vivo and may play a neuroprotective role in the survival of DA neurons. Our data might provide new evidence for the consideration of the immunoproteasome as a potential therapeutic target for PD.

\section{Additional files}

\section{Additional file 1: Figure S1. 6-Hydroxydopamine activates}

immunoproteasomes in DA neurons in a time-dependent manner. The expression of $\beta 5, \beta 5 i(A-B)$, TAP1 and Bip (C-D) in SN4741 cells after different durations of exposure to $200 \mathrm{nM} 6-\mathrm{OHDA} .{ }^{*} P<0.05$, compared with the normal condition. Data are presented as the mean $\pm S D ; n=4$; one-way ANOVA and post hoc SNK t-test. (TIF $518 \mathrm{~kb}$ )

Additional file 2: Figure S2. $\beta 5 i$ inhibition exacerbates 6 hydroxydopamine-induced damage in the striatum. (A) Immunostaining of TH in the rat striatum after 6-OHDA treatment for 4 weeks. Scale bar = $200 \mu \mathrm{m}$. (B) Quantification of TH immunoreactivity in the striatum. Data are presented as the mean $\pm S D ; n=4$ experiments; $* P<0.05$, compared with the control; \# $P<0.05$, compared with the 6-OHDA group; one-way ANOVA. (TIF $1137 \mathrm{~kb})$

\section{Acknowledgements}

This work was supported by research grants from National Key R\&D Program of China (2016YFC1306600, SQ2017YFSF110116), National Natural Science Foundation of China (81701254, 81471292, U1603281, U1503222, 81430021, 81501100, NO.8187050204), Science Foundation of Guangdong of China (2015A030311021, 2018A030313649), a technology project of Guangzhou
(201504281820463), Shandong Provincial Natural Science Foundation (BS2015YY041), International Project of Science and Technology for Guangdong (2016A050502025), Science and Technology of Guangdong of China (2013B022000026) and Collaborative Innovation Foundation of Guangzhou Science and Technology Bureau (2018-1202-SF-0019).

We thank Dr. Madhuvika Murugan, You-Sheng Xiao, Luan Chen, Li Zhang, Ming Lei, Chao-Hao Yang, Xiang Chen, Qin-Hui Huang, Wei-Guo Liu, LongJun Wu and Prof. Kai-Ping Li for their help to improve this manuscript. We thank Prof. Qian Yang provide SN4741 cells.

\section{Availability of supporting data}

All data mentioned in this article are available on published article.

\section{Ethical approval and consent to participate}

All procedures were approved by Institutional Animal Care and Use Committee of Guangzhou Medical University (NO.2017341).

\section{Funding}

National Key R\&D Program of China, National Natural Science Foundation of China, Science Foundation of Guangdong of China, Technology project of Guangzhou, Shandong Provincial Natural Science Foundation, International Project of Science and Technology for Guangdong, Science and Technology of Guangdong of China and the Collaborative Innovation Foundation of Guangzhou Science and Technology Bureau.

\section{Authors' contributions}

M-MS, H-JX and X-PY mainly conceived, designed study and drafted manuscript. The major experiments included the 6-OH DA model in vivo was performed by $\mathrm{L}-\mathrm{GH}$ and S-CC, and in vitro was performed by H-SX. Z-MM provided technical supports on in-cell western assay and color western blot, W-ZH and G-WY helped to the part on animal behavior and TH staining, Y-XL provided technical supports on LCM and gRT-PCR, Z-LM and W-L contributed to data analysis. Q-SG and C-CJ were involved in revising manuscript and experiment guide. Q-SG drew figures and tables, modified the manuscript. All authors read and approved final manuscript to be published. 


\section{Consent for publication}

Not applicable.

\section{Competing interests}

Authors have no conflict of interest to declare.

\section{Author details}

'Department of Neurology, First Affiliated Hospital of Guangzhou Medical University, Guangzhou 510120, Guangdong, China. 'Department of Neurology, Qilu Hospital of Shandong University, Jinan 250012, Shandong, China. ${ }^{3}$ Department of Neurology, First Affiliated Hospital of Sun Yat-sen University, Guangzhou 510080, Guangdong, China. ${ }^{4}$ Department of Neurology, Second Affiliated Hospital of Xinjiang Medical University, Urumchi 830011, Xinjiang, China. ${ }^{5}$ Clinic Brain Center, Guangzhou Hospital of Integrated Traditional and Western Medicine, Guangzhou 510800, Guangdong, China. ${ }^{6}$ Department of Blood Transfusion, Fifth Affiliated Hospital Southern Medical University, Guangzhou 510900, Guangdong, China. ${ }^{7}$ Department of Thoracic Surgery, First Affiliated Hospital of Guangzhou Medical University, Guangzhou 510120, Guangdong China.

\section{Received: 23 January 2018 Accepted: 30 July 2018}

\section{Published online: 17 August 2018}

\section{References}

1. Chu Y, Kordower JH. Age-associated increases of a-synuclein in monkeys and humans are associated with nigrostriatal dopamine depletion: is this the target for Parkinson's disease? Neurobiol Dis. 2007;25(1):134-49.

2. Taylor JM, Main BS, Crack PJ. Neuroinflammation and oxidative stress: COconspirators in the pathology of Parkinson's disease. Neurochem Int. 2013; 62(5):803-19.

3. Launay N, Ruiz M, Fourcade S, Schlüter A, Guilera C, Ferrer I, et al. Oxidative stress regulates the ubiquitin-proteasome system and immunoproteasome functioning in a mouse model of X-adrenoleukodystrophy. Brain. 2013; 136(3):891-904.

4. Bence NF, Sampat RM, Kopito RR. Impairment of the ubiquitin-proteasome system by protein aggregation. Science. 2001;292(5521):1552-5.

5. Wang J, Maldonado MA. The ubiquitin-proteasome system and its role in inflammatory and autoimmune diseases. Cell Mol Immunol. 2006;3(4):255-61.

6. Ciechanover A, Brundin P. The ubiquitin proteasome system in neurodegenerative diseases: sometimes the chicken, sometimes the egg. Neuron. 2003:40(2):427-46.

7. Dasuri K, Zhang L, Keller JN. Oxidative stress, neurodegeneration, and the balance of protein degradation and protein synthesis. Free Radic Biol Med. 2013;62:170-85.

8. Seifert U, Bialy LP, Ebstein F, Bech-Otschir D, Voigt A, Schröter F, et al. Immunoproteasomes preserve protein homeostasis upon interferoninduced oxidative stress. Cell. 2010;142(4):613-24.

9. Kincaid EZ, Che JW, York I, Escobar H, Reyes-Vargas E, Delgado JC, et al. Mice completely lacking immunoproteasomes show major changes in antigen presentation. Nat Immunol. 2012;13(2):129-35.

10. Chen S, Kammerl IE, Vosyka O, Baumann T, Yu Y, Wu Y, et al. Immunoproteasome dysfunction augments alternative polarization of alveolar macrophages. Cell Death Differ. 2016;23(6):1026.

11. Vilchez D, Saez I, Dillin A. The role of protein clearance mechanisms in organismal ageing and age-related diseases. Nat Commun. 2014:5(5):5659.

12. Mishto M, Bellavista E, Santoro A, Stolzing A, Ligorio C, Nacmias B, et al. Immunoproteasome and LMP2 polymorphism in aged and Alzheimer's disease brains. Neurobiol Aging. 2006;27(1):54-66.

13. Orre M, Kamphuis W, Dooves S, Kooijman L, Chan ET, Kirk CJ, et al. Reactive glia show increased immunoproteasome activity in Alzheimer's disease. Brain. 2013;136(5):1415-31.

14. Díaz-Hernández M, Martín-Aparicio E, Avila J, Hernández F, Lucas JJ. Enhaced induction of the immunoproteasome by interferon gamma in neurons expressing mutant huntingtin. Neurotox Res. 2004;6(6):463-8.

15. Mishto M, Raza ML, de Biase D, Ravizza T, Vasuri F, Martucci M, et al. The immunoproteasome $\beta 5 i$ subunit is a key contributor to ictogenesis in a rat model of chronic epilepsy. Brain Behav Immun. 2015;49:188-96.

16. Chen $X$, Zhang $X$, Wang $Y$, Lei $H$, Su H, Zeng J, et al. Inhibition of immunoproteasome reduces infarction volume and attenuates inflammatory reaction in a rat model of ischemic stroke. Cell Death Dis. 2015;6(1):e1626.
17. Bukhatwa S, Zeng B-Y, Rose S, Jenner P. A comparison of changes in proteasomal subunit expression in the substantia nigra in Parkinson's disease, multiple system atrophy and progressive supranuclear palsy. Brain Res. 2010;1326:174-83.

18. Nardo G, Trolese MC, Bendotti C. Major histocompatibility complex I expression by motor neurons and its implication in amyotrophic lateral sclerosis. Front Neurol. 2016;7:89.

19. Cebrián C, Zucca FA, Mauri P, Steinbeck JA, Studer L, Scherzer CR, et al. MHC-I expression renders catecholaminergic neurons susceptible to T-cellmediated degeneration. Nat Commun. 2014;5:3633.

20. Huang L, Xue Y, Feng DY, Yang RX, Nie T, Zhu G, et al. Blockade of RyRs in the ER attenuates 6-OHDA-induced calcium overload, Cellular HypoExcitability and Apoptosis in Dopaminergic Neurons. Frontiers in Cellular Neuroscience. 2017;11:52.

21. Zhang $S$, Gui $X-H$, Huang L-P, Deng $M-Z$, Fang R-M, Ke $X-H$, et al. Neuroprotective effects of $\beta$-asarone against 6-hydroxy dopamineinduced parkinsonism via JNK/BCl-2/Beclin-1 pathway. Mol Neurobiol. 2016;53(1):83-94.

22. Nijholt DA, De Kimpe L, Elfrink HL, Hoozemans JJ, Scheper W, et al. A selective inhibitor of the immunoproteasome subunit LMP7 blocks cytokine production and attenuates progression of experimental arthritis. Nat Med. 2009;15(7):781-7.

23. Ungerstedt U. Postsynaptic supersensitivity after 6-hydroxy-dopamine induced degeneration of the nigro-striatal dopamine system. Acta Physiol Scand. 1971;82(S367):69-93.

24. Goyal A, Pal N, Concannon M, Paul M, Doran M, Poluzzi C, et al. Endorepellin, the Angiostatic module of Perlecan, interacts with both the a2 $\beta 1$ integrin and vascular endothelial growth factor Receptor 2 (VEGFR2) a DUAL RECEPTOR ANTAGONISM. J Biol Chem. 2011;286(29):25947-62.

25. Hwang L-Y, Lieu PT, Peterson PA, Yang Y. Functional regulation of immunoproteasomes and transporter associated with antigen processing. Immunol Res. 2001;24(3):245-72.

26. Kummari E, Guo-Ross SX, Eells JB. Laser capture microdissection-a demonstration of the isolation of individual dopamine neurons and the entire ventral tegmental area. J Vi. Exp. 2015;96(96):e52336-e52336.

27. Fend F, Emmert-Buck MR, Chuaqui R, Cole K, Lee J, Liotta LA, et al. ImmunoLCM: laser capture microdissection of immunostained frozen sections for mRNA analysis. Am J Pathol. 1999;154(1):61-6.

28. Livak KJ, Schmittgen TD. Analysis of relative gene expression data using realtime quantitative $P C R$ and the 2- $\Delta \Delta C T$ method. methods. 2001:25(4):402-8.

29. Wang T, Yuan Y, Zou H, Yang J, Zhao S, Ma Y, et al. The ER stress regulator Bip mediates cadmium-induced autophagy and neuronal senescence. Sci Rep. 2016;6:38091.

30. Lawand M, Abramova A, Manceau V, Springer S, van Endert P. TAPdependent and-independent peptide import into dendritic cell Phagosomes. J Immunol. 2016;197(9):3454-63.

31. Lindå $\mathrm{H}$, Hammarberg $\mathrm{H}$, Piehl $\mathrm{F}$, Khademi $\mathrm{M}$, Olsson $\mathrm{T}$. Expression of $\mathrm{MHC}$ class I heavy chain and $\beta 2$-microglobulin in rat brainstem motoneurons and nigral dopaminergic neurons. J Neuroimmunol. 1999;101(1):76-86.

32. Ferrington DA, Hussong SA, Roehrich H, Kapphahn RJ, Kavanaugh SM, Heuss ND, et al. Immunoproteasome responds to injury in the retina and brain. J Neurochem. 2008;106(1):158-69.

33. Song S, Miranda CJ, Braun L, Meyer K, Frakes AE, Ferraiuolo L, et al. MHC class I protects motor neurons from astrocyte-induced toxicity in amyotrophic lateral sclerosis (ALS). Nat Med. 2016;22(4):397.

34. Díaz-Hernández M, Hernández F, Martín-Aparicio E, Gómez-Ramos P, Morán MA, Castaño JG, et al. Neuronal induction of the immunoproteasome in Huntington's disease. J Neurosci. 2003;23(37):11653-61.

35. Mo M-S, Huang W, Sun C-C, Zhang L-M, Cen L, Xiao Y-S, et al. Association analysis of proteasome subunits and transporter associated with antigen processing on Chinese patients with Parkinson's disease. Chin Med J. 2016; 129(9):1053.

36. Nijholt D, De Kimpe L, Elfrink H L, Hoozemans J JM, Scheper W. Removing protein aggregates: the role of proteolysis in neurodegeneration. Curr Med Chem. 2011;18(16):2459-76.

37. Brehm A, Krüger E. Dysfunction in protein clearance by the proteasome: impact on autoinflammatory diseases. Semin Immunopathol. 2015: Springer; 2015:323-33.

38. Reis J, Hassan F, Guan XQ, Shen J, Monaco JJ, Papasian CJ, et al. The immunoproteasomes regulate LPS-induced TRIF/TRAM signaling pathway in murine macrophages. Cell Biochem Biophys. 2011;60(1-2):119-26. 
39. Aiken $\mathrm{CT}$, Kaake RM, Wang $\mathrm{X}$, Huang L. Oxidative stress-mediated regulation of proteasome complexes. Molecular \& Cellular Proteomics. 2011;10(5):R110. 006924.

40. Unno M, Mizushima T, Morimoto Y, Tomisugi Y, Tanaka K, Yasuoka N, et al. The structure of the mammalian $20 \mathrm{~S}$ proteasome at $2.75 \AA$ resolution. Structure. 2002;10(5):609-18.

41. Aso E, Lomoio S, López-González I, Joda L, Carmona M, Fernández-Yagüe N, et al. Amyloid generation and dysfunctional immunoproteasome activation with disease progression in animal model of familial Alzheimer's disease. Brain Pathol. 2012;22(5):636-53.

42. Cheroni C, Marino M, Tortarolo M, Veglianese P, De Biasi S, Fontana E, et al. Functional alterations of the ubiquitin-proteasome system in motor neurons of a mouse model of familial amyotrophic lateral sclerosist. Hum Mol Genet. 2009;18(1):82-96.

43. Pickering AM, Koop AL, Teoh CY, et al. The immunoproteasome, the $20 \mathrm{~S}$ proteasome and the PA28a $\beta$ proteasome regulator are oxidative-stressadaptive proteolytic complexes. Biochem J. 2010;432(3):585-95.

44. Nathan JA, Spinnenhirn V, Schmidtke G, et al. Immuno-and constitutive proteasomes do not differ in their abilities to degrade ubiquitinated proteins. Cell. 2013;152(5):1184-94.

45. Haroon N, Maksymowych WP, Rahman P, Tsui FW, O'Shea FD, Inman RD. Radiographic severity of ankylosing spondylitis is associated with polymorphism of the large multifunctional peptidase 2 gene in the Spondyloarthritis research consortium of Canada cohort. Arthritis Rheum. 2012;64(4):1119-26.

46. Mercado G, Castillo V, Vidal R, Hetz C. ER proteostasis disturbances in Parkinson's disease: novel insights. Front Aging Neurosci. 2015;7:39.

47. Ugunklusek A, Tatham MH, Elkharaz J, Constantinteodosiu D, Lawler K, Mohamed $\mathrm{H}$, et al. Continued $26 \mathrm{~S}$ proteasome dysfunction in mouse brain cortical neurons impairs autophagy and the Keap1-Nrf2 oxidative defence pathway. Cell Death Dis. 2017;8(1):e2531.

48. Ebstein F, Kloetzel P-M, Krüger E, Seifert U. Emerging roles of immunoproteasomes beyond MHC class I antigen processing. Cell Mol Life Sci. 2012;69(15):2543-58.

49. Askanas V, Engel WK. Inclusion-body myositis a myodegenerative conformational disorder associated with $A B$, protein misfolding, and proteasome inhibition. Neurology. 2006;66(1 suppl 1):S39-48.

50. Ryu EJ, Harding HP, Angelastro JM, Vitolo OV, Ron D, Greene LA. Endoplasmic reticulum stress and the unfolded protein response in cellular models of Parkinson's disease. J Neurosci. 2002;22(24):10690-8.

51. Tao K, Wang B, Feng D, Zhang W, Lu F, Lai J, et al. Salidroside protects against 6-Hydroxydopamine-induced cytotoxicity by attenuating ER stress. Neurosci Bull. 2016;32(1):61-9.

52. Basler M, Mundt S, Muchamuel T, Moll C, Jiang J, Groettrup M, et al. Inhibition of the immunoproteasome ameliorates experimental autoimmune encephalomyelitis. EMBO Mol Med. 2014;6(2):226-38. e201303543

53. Shastri N, Nagarajan N, Lind KC, Kanaseki T. Monitoring peptide processing for MHC class I molecules in the endoplasmic reticulum. Curr Opin Immunol. 2014;26:123-7.

54. McDermott A, Jacks J, Kessler M, Emanuel PD, Gao L. Proteasome-associated autoinflammatory syndromes: advances in pathogeneses, clinical presentations, diagnosis, and management. Int J Dermatol. 2015;54(2):121-9.

55. McDermott A, de Jesus AA, Liu Y, Kim P, Jacks J, Sanchez GAM, et al. A case of proteasome-associated auto-inflammatory syndrome with compound heterozygous mutations in PSMB8. J Am Acad Dermatol. 2013;69(1):e29.

56. Basler M, Mundt S, Bitzer A, Schmidt C, Groettrup M. The immunoproteasome: a novel drug target for autoimmune diseases. Clin Exp Rheumatol. 2015;33:74-9.

57. Mangold CA, Masser DR, Stanford DR, Bixler GV, Pisupati A, Giles CB, et al. CNS-wide sexually dimorphic induction of the major histocompatibility complex 1 pathway with aging. J Gerontol A Biol Sci Med Sci. 2016;72(1): 16-29. glv232

58. McGeer P, Itagaki S, Boyes B, McGeer E. Reactive microglia are positive for HLA-DR in the substantia nigra of Parkinson's and Alzheimer's disease brains. Neurology. 1988;38(8):1285.

59. Meuth SG, Herrmann AM, Simon OJ, Siffrin V, Melzer N, Bittner S, et al. Cytotoxic CD8+ T cell-neuron interactions: perforin-dependent electrical silencing precedes but is not causally linked to neuronal cell death. J Neurosci. 2009;29(49):15397-409.

60. Neumann $\mathrm{H}$, Cavalie A, Jenne DE, Wekerle H. Induction of MHC class I genes in neurons. Science. 1995;269(5223):549.

Ready to submit your research? Choose BMC and benefit from:

- fast, convenient online submission

- thorough peer review by experienced researchers in your field

- rapid publication on acceptance

- support for research data, including large and complex data types

- gold Open Access which fosters wider collaboration and increased citations

- maximum visibility for your research: over $100 \mathrm{M}$ website views per year

At $\mathrm{BMC}$, research is always in progress.

Learn more biomedcentral.com/submissions 